was obtained from which the products described by Evans, Emerson and Emerson ${ }^{1}$ could be isolated, in addition to a crystalline allophanate, m.p. $70^{\circ}$, which has not yet been examined biologically.

The above purified oil from the rice concentrate deposited on standing a crystalline substance, m.p. $73^{\circ}$, which appears to be an aliphatic mono-unsaturated alcohol containing approximately 20 carbon atoms. After removal of this alcohol, the oil on saturation with cyanic acid in benzene solution gave a complex mixture of substances from which a small amount of an allophanate, m.p. $135^{\circ}-138^{\circ}$, having the properties of $\beta$-tocopheryl allophanate ${ }^{1}$, could be isolated together with a larger quantity of an allophanate, m.p. $195^{\circ}-200^{\circ}$; the parent alcohol of the latter substance is being tested biologically.

In view of the formula of the tocopherols and the probability that they may represent mono-alkyl ethers of durohydroquinon $e^{6}$, the occurrence in active rice-germ oil concentrates of the alcohol m.p. $73^{\circ}$ may be of some significance. The work on the structure of these various compounds, of which fuller details will be published elsewhere, is being continued.

$\begin{array}{cl}\text { Biochemical Department, } & \text { A. R. ToDD. } \\ \text { Lister Institute, } & \text { H. BERGEL. } \\ \text { London. } & \text { T. S. Word }\end{array}$
July 29.

1 Evans, Emerson and Emerson, J. Biol. Chem., 113, 319 (1936).

${ }^{2}$ Emerson, Emerson and Evans, Science, 88, 421 (1936).

${ }^{3} \mathrm{Kimm}$, J. Agric. Chem. Soc. Jap., 11, 514 (1935).

'Kimm, Sci. Pap. Inst. Phys. Chem. Res. Tokyo, 28, 74 (1935).

- Karrer and Salomon, Helv. chim. Acta., 20, 424 (1937).

- Fernholz, J. Amer. Chem. Soc., 59, 1154 (1937).

\section{Para-Cresol from the Urine of Pregnant Mares}

Burrows, Cook et al. ${ }^{1}$ have recently isolated $p$-cresol, along with certain ketones of the sterol group, from the urine of a man with a malignant tumour of the adrenal cortex. The urine $(301$.$) was$ submitted to an initial hydrolysis by boiling with hydrochloric acid, and $0.75 \mathrm{gm}$. of $p$-cresol was isolated from the non-ketonic phenol fraction.

It should be recorded that in this Laboratory a similar observation has been made in regard to the urine of pregnant mares. Approximately $110 \mathrm{gm}$. of pure $p$-cresol, containing no detectable trace of $o$ - or $m$-isomeride, was obtained from 400 gallons of mares' urine, which had been submitted to hydrolysis -a considerably higher proportion than that reported by Burrows, Cook et al. for a pathological specimen of human male urine. The significance of this finding is not yet apparent.

$$
\text { P. G. Marshall. }
$$

Biochemical Laboratory, The British Drug Houses, Ltd.,

Graham Street, London, N.1. July 29.

B urrows, Cook, Roe and Warren, Biochem. J., 31, 950 (1937).

\section{Artificial Release of Crossing-over in Meiosis and Mitosis}

THe experiments with artificial production of crossing-over described below belong to a new series which were finished in the spring of this year; they enable us to carry our analysis much further and bring out a series of facts which are entirely new. The following are the main conclusions drawn from this work :
1. (a) X-ray treatment of Drosophila melanogaster males induces not only spermatogonial, but also meiotic crossing-over.

(b) Histological examination of irradiated testes revealed that the early spermatocytes of the first order possess the greatest susceptibility to treatment, whereas the spermatogonia, though also affected, react to a much less extent.

2. (a) The mitotic origin of spermatogonial crossing. over induced by X-rays in Drosophila melanogaster has been definitely established.

(b) The presence of inversions lowers somewhat the frequency of spermatogonial crossing-over throughout the non-inverted region of chromosome III. This proves that there is a similarity between regular meiotic and induced mitotic crossing-over.

(c) The frequency of spermatogonial crossing-over is extremely high in the central region (which carries the spindle fibre) of chromosome III. In the remain. ing regions of the chromosomes there is a closer correspondence with the cytological map than in the case of the normal meiotic crossing-over in females.

(d) The frequency of induced crossing-over is higher for later than for earlier spermatogonial stages.

(e) The frequeney of spermatogonial erossing-over is not proportional to the X-ray dose (a comparison at $1,000 \mathrm{r}$. and $4,000 \mathrm{r}$. shows a frequency greater than fourfold in the latter case).

3. (a) No oogonial crossing-over was released in female larvæ of Drosophila melanogaster with a treatment of $2,000 \mathrm{r}$.

(b) The $\frac{C y}{b c n c}$ females used in this experiment showed nevertheless a statistically significant increase in crossing-over between ' $b$ ' and ' $c n$ '. Since only the gonial stages were irradiated, this result must be taken to mean that there exists an after-effect of the treat. ment on crossing-over.

4. (a) Contrary to the current point of view, which excludes the possibility of crossing-over in the late oocyte of Drosophila melanogaster, we were successful in inducing interchange at that stage; this type of interchange we designate as late meiotic crossing. ovier.

(b) This fact raises certain difficulties for Darlington's and Belling's points of view on the mechanism of crossing-over, whereas it favours the interpretation offered by Sax.

From the above summarized results we may draw the following general conclusions :

(1) Crossing-over is a feature to some extent inherent in both meiosis and mitosis.

(2) The absence of regular crossing-over in the gonial stages of gametogenesis in Drosophila melanogaster can be explained by the disadvantages somatic crossing-over would offer ; the suppression of crossingover in the soma means its suppression in mitosis and also in the gonial cells.

(3) The hypothesis of Haldane and Huxley, which is based on the disadvantages that crossing. over would offer in the heterogametic sex, does not sufficiently substantiate the nearly complete absence of normal crossing-over in meiosis of Drosophila melanogaster males.

A detailed account of the work will appear in Russian (with an extensive German summary) in the Biologichesky Zhurnal.

Institut of Experimental Biology,

Heinrich Friesen. Moscow. July 13. 